\title{
Development of Selective Chemical Gas Sensors Based on Novel Polymeric Phthalocyanines
}

Ivanova/Olga, Chuprin/Maxim, Tarasova/Alla, Shevchenko/Anatoly, Krutovertsev/Sergey,

Sherle/Alla*, Oleinik/Eduard*

JSC "Ecological sensors and systems"

P/B 146, Zelenograd, Moscow, 124460, Russia

Institute of Chemical Physics of RAS

ul. Kosygina 4, Moscow, 117977, Russia

\section{INTRODUCTION}

The properties and applications of phthalocyanine $(\mathrm{Pc})$ and its metall derivatives are studied extensively [1-3]. In the recent years special interest attracts to phthalocyanine films in regards with their application in gas sensors. This fact is due to the outstanding features distinguishing these substances from the multitude of other groups of macrocyclic complexes in that they are aromatic macrocycles with a unique conjugated $\pi$-system, which determines their chemical and thermal resistance, semiconductor properties and biological activity. It is known that the surface of phthalocyanine films can adsorb molecules of gases to change of physicochemical and optical properties of Pcs. The physicochemical properties (conductivity) and optical characteristics (absorption spectrum) of novel synthesized polyphthalocyanines (PPc), containing $\mathrm{Zn}, \mathrm{Cu}, \mathrm{Mn}, \mathrm{Fe}, \mathrm{Pb}$ were investigated.

\section{EXPERIMENTAL}

The conductivity of the films, their resistance-temperature relationship, sensor properties relative to gases as well as effect of the conditions of film formation on their characteristics were taken into account upon choice of method signal registration and construction developing of the PPc based sensors.

The properties of the sensor were examined on sample gas mixtures using a dynamic blender "Environics-4000" (Environics, USA); the Dräger test ampoules (Dräger, Germany) were employed. All measurements were made at the same humidity and different temperature.

The substances (fig.1) were synthesized by polycyclotetramerization of tetranitrile of pyromellitic acid in bulk and in polar solvents at $180-300^{\circ} \mathrm{C}$ for $5-30$ hours in the presence of $0-5 \mathrm{~mol} \%$ carbamide.

The films of PPc were sputtered in vacuum or precipitated from the solutions. The films thermal sputtering were obtained in vacuum using UVN-71P3 plant at evaporation temperature of $300-1100^{\circ} \mathrm{C}$ and pressure in chamber $1,33 \times 10^{-8}$ bar. The evaporator-to-base distance was $100 \mathrm{~mm}$. To stabilize the film properties those were annealed in air at $100-250^{\circ} \mathrm{C}$.
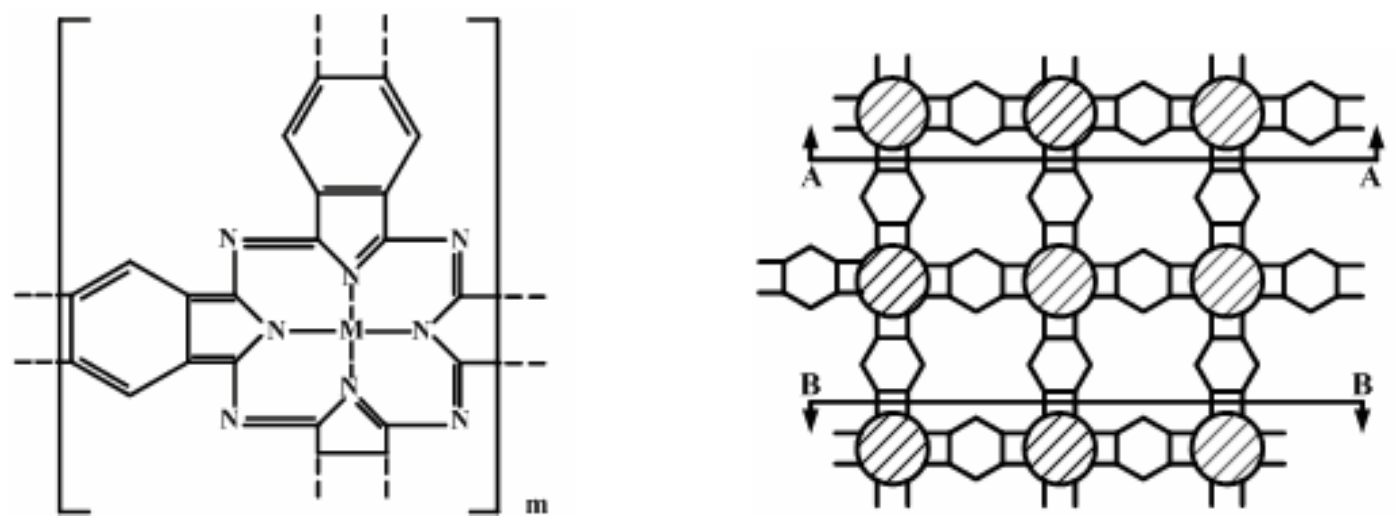

$\mathrm{M}=\mathrm{H}_{2}, \mathrm{Zn}, \mathrm{Fe}, \mathrm{Cu}, \mathrm{Co}, \mathrm{Ni}, \mathrm{Mn}$

Fig.1 Typical structure of polyphthalocyanine (oligophthalocyanine - inside profile AA-BB). 

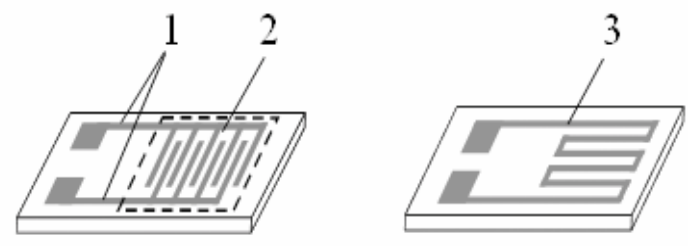

Fig. 2 Test structure for measurements:

1 - phthalocyanine film; 2-interdigitated electrodes; 3-heater.

The films were deposited on tested structures and glass substrates. Test structures were formed on dielectric (sitall) substrate, with dimensions 15,0x5,0 mm. Two comb-like electrodes, made of nickel, were located on one side of the substrate; the opposite side contained nickel film heater. The active area with the comb-like electrodes and the heater on the opposite side was $4 \times 8 \mathrm{~mm}$ in size (fig.2).

The investigations were carried out under the sensor thermal stabilization conditions in the range $50-250^{\circ} \mathrm{C}$. Sensor heating, conductivity and resistance measurements were maintained by two 16-channel measurement units and IBM PC program that operates in real time. Up to 32 sensors are able to be placed in the measurement chamber. The device has function to maintain a stable sensor temperature in the range $30-500^{\circ} \mathrm{C}$ with accuracy $1{ }^{\circ} \mathrm{C}$. The developed measurement unit allows to conduct simultaneously measurements of resistance of gas-sensitive layers on 32 channels in the range $500 \mathrm{Ohm}-1,5 \mathrm{GOhm}$ with accuracy $2,5 \%$. Besides that, the measurement unit regulates and controls sensor heating processes during the measurements.

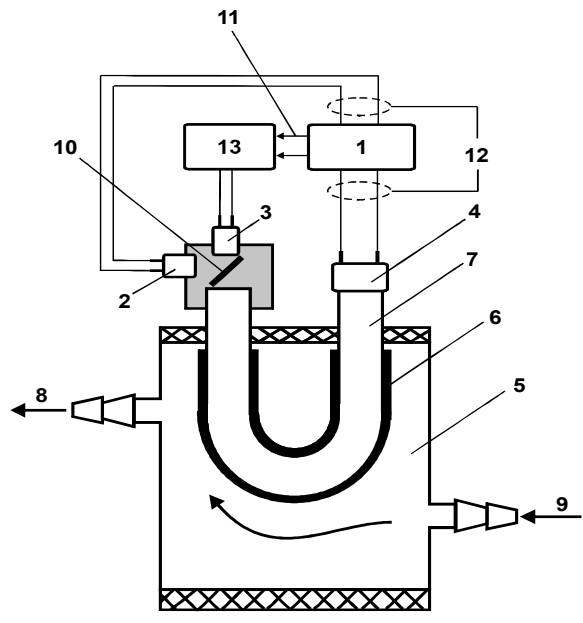

Fig.3 The design of a flow-through optical sensor: 1 - the measuring block ; 2 - the reference photo receiver; 3 - the LED; 4 - the measuring photo receiver; 5 - the flow-through measurement cell; 6 the film; 7 - the U-shaped cylindrical waveguide with film; 8, 9 - the input and output tubes; 10 - the beam-splitting system; 11 - the feedback channel; 12 - the channels of measuring; 13 - the current source.

An optical sensor consists of sensitive element and measuring block (fig. 3). The physical principle of the sensor sensitive element action is based on the dependence of light loss from environment refraction index. The surface of sensitive element was covered with phthalocyanine films by aid of Langmuir - Blodgett method which allows obtaining very thin "molecular-smooth" films of a given thickness.

The sensitive element is placed in a flow-through measuring cell (fig.1) and represents a cylindrical optical waveguide made of silicon. It is $2.5 \mathrm{~mm}$ in diameter and arched to 180 degrees. The arch radius is $2.5 \mathrm{~mm}$ (7). A light transmitter which divides a light beam according to ratio 1:1, a light source and a reference photo receiver (a photo diode) (2) are placed at the input end of the waveguide. A LED with the wavelength of $660 \mathrm{~nm}$ as the light source (3) was used for this experiment, because there is absorption maximum for phthalocyanine at this wavelength. The reference photo receiver is athwart to the axis of the light source. A measuring photo receiver is fixed at the output end of the 
waveguide. The measuring block contains a specially designed microprocessor unit which was calculated results of measurements.

The signal magnitude of the sensor was determined by the equation:

$U=\frac{U_{i}-\bar{U}_{i, b}}{U_{0}-\bar{U}_{0, b}}$,

(1)

where $U_{i}, U_{0}$ - value of the signal in measuring channel and in reference channel correspondingly, $\bar{U}_{i, b}, \bar{U}_{0, b}$ - average value of the background signal in measuring channel and in reference channel correspondingly.

The sensor relative signal was determined by the equation:

$U_{r}=\frac{U-U_{\text {air }}}{U_{\text {air }}}$

(2)

where $U_{\text {air }}$ - signal of the sensor in air.

\section{RESULTS}

The typical characteristics of the PPc films at the influence of ammonia are shown on fig.4. The film resistance in the analyzed medium is seen to increase monotonically with the $\mathrm{NH}_{3}$ concentration. However the sensitivity range for FePPc is narrow and corresponds to gas micro concentrations. At the same time (fig.5) resistance of the PPc films decrease at the presence NO in air. This fact is interesting for realization of sensors based on PPc films as it permits to increase the selectivity of determination.

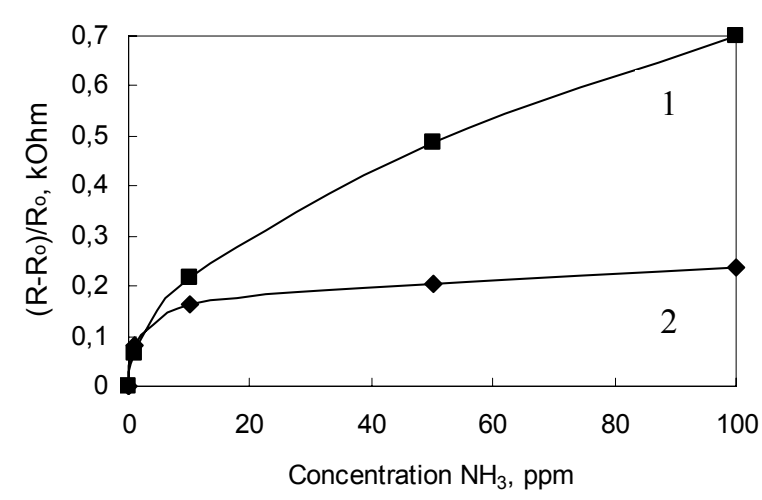

Fig.4 Typical $\mathrm{NH}_{3}$ concentration dependence of resistance for PPc films $\left(100^{\circ} \mathrm{C}\right)$ :

1-FePPc; 2- (Zn-Cu)PPc.

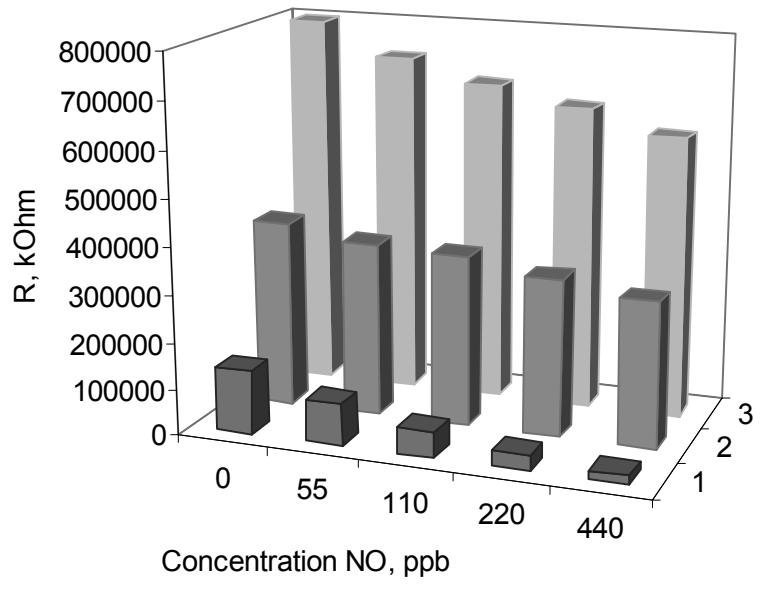

Fig.5 Typical NO concentration dependence of resistance for $\mathrm{PPc}$ films $\left(100^{\circ} \mathrm{C}\right)$ : 1-(Zn-Cu)PPc; 2- CoPPc; 3- FePPc.

Change of electro-physical characteristics of CuPPc at the interaction with $\mathrm{NO}_{2}$ micro concentrations is accompanied by optical changes of substance. The typical behaviour of PPc films in the presence $\mathrm{NO}_{2}$ in the measuring air is shown on fig.6. The advantage of the optical method (fig.7) consists in possibility of measurement at room temperature as all electro-physical changes are implemented at increased temperature. Moreover films have high resistance and it makes difficulties for measurement. 


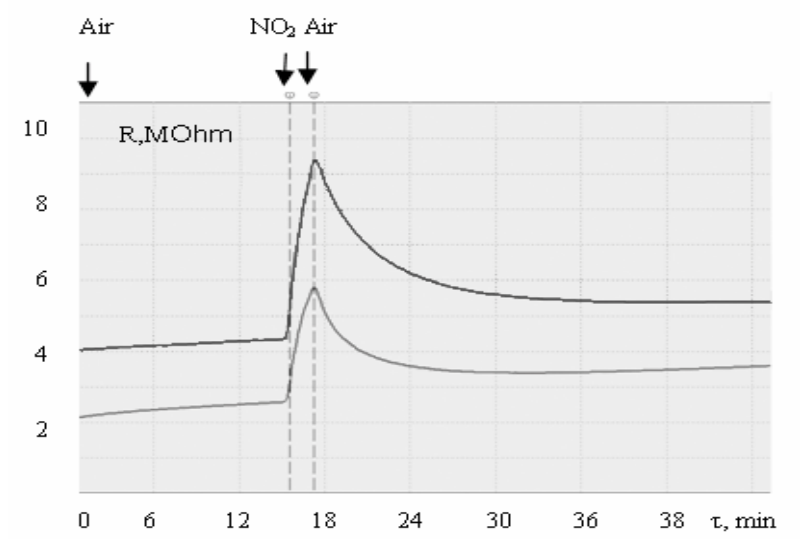

Fig. 6 Resistance change of PPc at $1,9 \mathrm{ppb} \mathrm{NO}$ and $180^{\circ} \mathrm{C}$ :

1 -zinc oligophthalocyanine;

2-copper polyphthalocyanine.

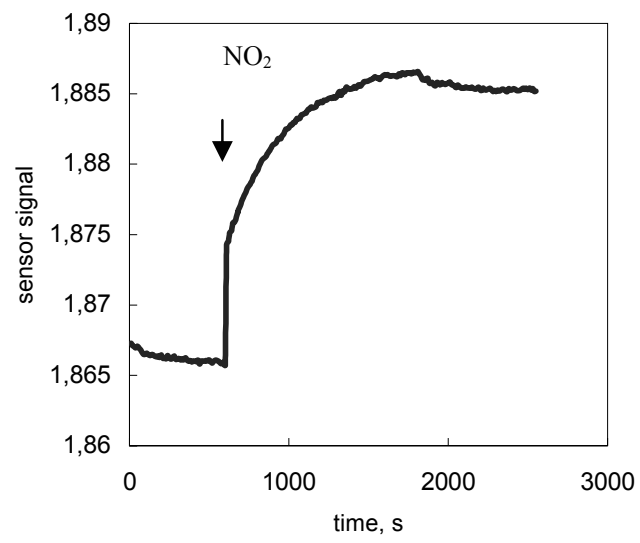

Fig. 7 Optical signal of copper PPc at $1,9 \mathrm{ppb} \mathrm{NO}$ and room temperature.

The measurements were run for the various thicknesses of films and temperature. Optical sensor signal was determined on 400 and $600 \mathrm{~nm}$. It was found, that PPc of Cu has high sensitivity to $\mathrm{NO}_{2}$ micro concentrations and $\mathrm{H}_{2} \mathrm{~S}$. The sensitivity increases with growth of $\mathrm{Cu}$ content in substance. It was shown, that PPc of Mn can be utilized as sensitive material to detect $\mathrm{O}_{2}$ concentration change. The investigated materials have satisfactory sensitivity to different gases: PPc of $\mathrm{Fe}$ and $\mathrm{Pb}$ can be used as sensitive layers in gas $\mathrm{NO}_{2}$ and $\mathrm{H}_{2} \mathrm{~S}$ sensors. Besides, PPc of $\mathrm{Fe}$ demonstrated reversible signal change to $\mathrm{NH}_{3}$ in range $0-100 \mathrm{ppm}$. It is important, that the character of resistive sensor signal change is determined by PPc conductivity type for all examined materials and corresponds with optical sensor signal in most cases.

\section{REFERENCES}

[1] W. Hu, Y. Liu, Y. Xu, S. Liu, S. Zhou, P. Zeng, D.B. Zhu: Sensors and Actuators, B 56, 1999, p.228

[2] Kuo-Chuan Ho, Yi-Ham Tsou: Sensors and Actuators, B 77, 2001, p.253

[3] E. van Faassen, H. Kerp: Sensors and Actuators, B 88, 2003, p.329 